\title{
The value of a screen and treat policy for Chlamydia trachomatis in women attending for termination of pregnancy
}

Tamsin M Groom, MB BS, MRCOG, Specialist Registrar; Peter Stewart, BM BcH, FRCOG, Consultant Obstetrician and Gynaecologist; Heike Kruger, MB BS, Senior House Officer, Department of Obstetrics and Gynaecology, Northern General Hospital, Sheffield, UK

Gill Bell, SRN, Health Advisor, Department of Genitourinary Medicine, Royal Hallamshire Hospital, Sheffield, UK.

Correspondence: Mr P Stewart, Consultant Obstetrician and Gynaecologist, Department of Obstetrics and Gynaecology, Northern General Hospital NHS Trust, Herries Road, Sheffield, S5 7AU, UK.

(Accepted September $11^{\text {th }}, 2000$ )

\section{Summary}

We aimed to assess the efficacy of a screen and treat policy for sexually transmitted infections in women requesting termination of pregnancy, with particular reference to Chlamydia trachomatis. A retrospective review of 100 consecutive cases of Chlamydia-positive women between December 1995 and February 1998, was performed. The referral rate to genitourinary medicine (GUM), the subsequent management, contact tracing and treatment of partners were assessed.

Ninety-nine women were referred to the GUM department, of whom 72 (71.4\%) attended. Eighteen (25\%) required further treatment. Seventy-five women identified 89 potentially infected men, of whom 62 (69\%) attended for treatment.

Identification of positive cases with referral to GUM enabled tests of cure to be carried out, treatment of those reinfected and re-enforcement of behaviour modification, in addition to successful contact tracing. This policy may confer greater benefit for the patient herself and make a significant impact on the reservoir of infection in the community when compared to a policy of blanket prophylactic antibiotic treatment at the time of termination. The implications for future service provision are discussed.

Key words

Chlamydia trachomatis, GUM, screen and treat

\section{Key message points}

- $71 \%$ of women who are Chlamydia-positive attended GUM when referred.

- Despite advice and treatment, $25 \%$ had been exposed to re-infection before they attended.

- $96 \%$ of those male partners contacted were Chlamydia-positive or were potentially infected.

- Contact tracing may help to reduce the reservoir of infection in the community.

\section{Introduction}

Chlamydia trachomatis is a common cause of pelvic infection. If untreated it is estimated that more than a third of women will develop salpingitis. Even when treated $17 \%$ of women with pelvic inflammatory disease (PID) will become infertile; an equal number will have chronic pelvic pain and $10 \%$ with a past diagnosis of PID will have an ectopic pregnancy. Rates of postabortal PID range from 5 to $10 \% .1,2$
In Sheffield all women attending for termination of pregnancy (TOP) are screened for sexually transmitted infections. Those positive for Chlamydia or Gonorrhoea are treated and referred to GUM for test of cure and contact tracing.

This study was designed to assess the efficacy of our current policy. An alternative practice using prophylactic antibiotics for all women undergoing TOP has, however, been recommended by others. ${ }^{3}$

\section{Method}

The screen and treat policy at our hospital has been in place for 12 years. All women attending for TOP have endocervical and high vaginal swabs taken either at the family planning clinic, by their GPs, or at the hospital. Screening is performed for Chlamydia Trachomatis, Gonococcus, Ureaplasma urealyticum and Mycoplasma on endocervical swabs. Vaginal pathogens are isolated from high vaginal swabs. Chlamydia is detected by enzyme immuno assay and verified by direct immunofluorescence at a centralised public health laboratory. Those found to be Chlamydia-positive are given a 2 week course of triple tetracycline (Deteclo, Ledearle) $300 \mathrm{mg}$ bd., together with an information leaflet emphasising the asymptomatic nature of the disease, the sequelae of untreated and repeated infections, and the importance of compliance and partner participation. All women are referred to the GUM department for follow-up, unless strong objections are voiced. The referrals are arranged by the clinical nurse specialist for the TOP service using agreed referral protocols. The woman's details are given to the GUM department, who then send an appointment. If the patient fails to attend, the health advisor from GUM uses standard procedures to try to make contact.

One hundred consecutive cases of Chlamydia between December 1995 and February 1998 were identified and the notes obtained from medical records. A proforma was designed and details of the index admission, treatment, and referral were obtained from the notes. The proforma was sent to the GUM department where it was anonymised and information regarding partners, treatment of both parties, compliance and re-exposure was obtained.

Results

The current prevalence rate of Chlamydial infection among our population of women requesting TOP is $3.6 \%$. 
Characteristics of women studied.

The mean age of the women studied was 22 years; the Mode was 19; two patients were under 15 and the oldest was 39 . The majority (81) of the women were referred for TOP from the central family planning clinic, 18 were referred from their general practitioners (GPs), and one was a hospital inpatient. Forty-nine women underwent a surgical TOP, 49 underwent a medical TOP, one woman decided to continue with her pregnancy, and one miscarried prior to her termination.

\section{Infections identified}

Forty-two women had Chlamydia alone. Fifty-two were found to have other infections in addition to Chlamydia. Six were equivocally positive for Chlamydia (positive on enzyme immunoassay but not confirmed by direct immunofluorescence). Several women had other infections as indicated in Table 1.

Table 1 Infections identified other than Chlamydia

\begin{tabular}{lc}
\hline Type of Infection & No. of women affected \\
\hline Mycoplasma & 27 \\
Ureaplasma & 26 \\
Bacterial vaginosis & 16 \\
Candida & 11 \\
Gonorrhoea & 5 \\
Trichomonas & 1 \\
\hline
\end{tabular}

\section{Attendance at GUM}

Ninety-two new cases were referred to GUM, and seven were already under their auspices pre-TOP, giving a referral rate of $99 \%$. Sixty-five of the 'new' referrals attended, giving a total number attending of $72(71.4 \%)$.

Of the 72 women attending for follow-up, 18 (25\%) required further treatment: 16 had been re-exposed to infection, one was non-compliant with antibiotic treatment secondary to a gastro-intestinal upset and one failed to collect her antibiotics from the ward. A further three women were contacted by telephone, they declined to attend themselves, but provided contact information.

\section{Partner participation}

Seventy-five women identified 89 partners, of whom 62 attended GUM (Table 2). Seven of the men were traced following confidential telephone enquiries by health advisors and attended while their female counterparts did not. Of these 62 men, 27 had proven genitourinary infections, and 33 had epidemiological evidence of Chlamydial contact (i.e. these men were known to have had sexual contact with an infected woman but Chlamydia was not isolated at screening) (Table 3).

Table 2 Contact tracing

\begin{tabular}{cccc}
\hline $\begin{array}{c}\text { No. Male partners } \\
\text { identified in } \\
\text { previous 6 months }\end{array}$ & $\begin{array}{c}\text { No. Women } \\
\text { identifying male } \\
\text { partners in previous } \\
6 \text { months }\end{array}$ & $\begin{array}{c}\text { No. Male } \\
\text { partners seen }\end{array}$ & $\begin{array}{c}\text { \% Male } \\
\text { partners seen }\end{array}$ \\
\hline 1 & 63 & 47 & 74.6 \\
2 & 11 & 14 & 63.6 \\
4 & 1 & 1 & 25 \\
\hline
\end{tabular}

\section{Treatment of partners}

In addition to those with proven Chlamydial infection or non-specific urethritis (NSU), 33 men received treatment on epidemiological grounds. Fifty-six men were therefore treated with antibiotics appropriate for the eradication of
Table 3 Diagnoses in male partners attending GUM

Chlamydia

Non-specific urethritis

'Complicated' NSU

Gonorrhoea

Ano-genital warts

Scabies

Primary genital herpes

Epidemiological evidence of Chlamydial infection only

Chlamydial infection. A further two men had already been treated by their GPs. Therefore $93.5 \%$ (58) men received treatment for proven or suspected Chlamydial infection. Four men did not: one left the department prior to leaving a specimen of urine and one was documented as having 'no signs of infection'. It was unclear from the records why the remaining two were not treated.

\section{Discussion}

Chlamydia is a treatable sexually transmitted infection (STI) with serious sequelae including pelvic inflammatory disease, chronic pelvic pain, ectopic pregnancy, tubal factor infertility and neonatal infections. It is also the most common STI in the UK and has been found to be asymptomatic in up to $70 \%$ of infected women in the developed world. ${ }^{4}$ Young women undergoing TOP are known to be particularly at risk, as are young women in general, single nulliparous women, those on the oral contraceptive pill, those who use no contraception at all, and those with two or more sexual partners in the last 12 months. ${ }^{2}$

Prevalence rates amongst women attending for TOP in the UK vary between $5 \%$ and $12 \% .^{3,5-8}$ At the time of this study our prevalence rate was only $3.6 \%$, which is lower than a previous audit in $1989^{9}(8.3 \%)$. This reduction may reflect a successful policy of referral to GUM and a subsequent decrease in rates of infection within the community as a whole, though this is unlikely to have been entirely responsible for this particularly low prevalence rate. Another possible explanation is that in 1989 all patients were seen in hospital allowing consistent techniques for swab taking and rapid specimen transfer to the laboratories. With an increased variety of medical staff taking swabs during the study period there is scope for inaccuracies in swab taking techniques in addition to delayed transfer of specimens to the laboratory.

Nucleic acid amplification techniques, such as PCR assays and ligase chain reaction tests, improve the detection rate of Chlamydia trachomatis infection. The implementation of such techniques may increase the identification rate of positive cases.

Despite both verbal and written information given to all patients in our study regarding the serious implications of Chlamydial infection, only $71 \%$ attended for follow-up at GUM and $25 \%$ required further treatment. The use of a single dose of azithromycin rather than a 2 week course of tetracycline may aid compliance with the antibiotic regime, but the rate of re-infection prior to attendance at GUM is concerning. One patient was not referred to GUM, emphasising that even in a well-established service there is room for improvement in communication between health care agencies.

Penney et al advocate the routine use of prophylactic antibiotics in all women attending for TOP. ${ }^{3}$ In their study 45 Chlamydia-positive women were identified in their screen and treat group. They were all offered GUM followup. However, only 11 (24\%) attended, identifying only 10 partners of whom only four $(40 \%)$ attended. 
To attain a minimum standard of contact tracing it has been recommended that at least $70 \%$ of index patients should have at least one contact attending GUM. ${ }^{10}$ In our study $75(74 \%)$ women (three of whom did not attend themselves but were contacted by telephone) identified 89 contacts, of whom $62(70 \%)$ attended. This gives a high contact-per-patient rate of $82.6 \%$. The Scottish study falls far short of this at $36 \%$ and it is perhaps not surprising that they were therefore unable to recommend such a policy. Contact tracing and follow-up is known to be more effective in certain groups such as those with steady partners, and less so in others including commercial sex workers, casual sexual encounters, young partners, and ethnic minority groups. ${ }^{11}$ It does, however, aim to prevent re-infection. It can encourage behaviour change, diminish the reservoir of infection in the community and thereby interrupt the transmission chain. ${ }^{12}$ These benefits are denied to patients offered prophylactic antibiotics alone.

A possible alternative would be patient-delivered partner medication (PDPM). This method is used more commonly in Scandinavia, but was recently considered in an American paper, ${ }^{13}$ which found PDPM to significantly reduce the reinfection rate when compared to contact tracing (11.5\% vs. $25.5 \%$ ) in two groups of young women attending a family planning clinic. Attendance at GUM, however, does afford further opportunities for education on avoidance of reinfection, and potential behaviour modification in this vulnerable group. Our results emphasise the inadequacy of a prophylactic blanket treatment regime, as many of these patients would simply return to the reservoir of infection in the community.

We conclude that our study shows the relative success of a screen and treat policy, together with the value of referral to GUM departments. More effective identification of Chlamydia-positive patients is required, and even greater emphasis needs to be placed on informing women of the potential seriousness of this infection.

\section{Statements on funding and competing interests \\ Funding. None.}

Competing interests. None declared.

References

Penney GC. Prophylactic antibiotic therapy for abortion. In: Templeton A, editor. The Prevention of Pelvic Infection. London: RCOG Press, 1996: 211-222.

CMO's Expert Advisory Group. Chlamydia trachomatis. London: Department of Health, 1998.

Penney GC, Thomson M, Norman J, et al. A randomised comparison of strategies for reducin infective complications of induced abortion. Br J Obstet Gynaecol 1998; 105: 599-604. sequelae Am J Obstet Gynecol 1991; 164 (6): 1771-1781.

Duthie S, Hobson D, Tait I, et al. Morbidity after termination of pregnancy in the first trimester. Genitourin Med 1987; 63: 182-187.

Morton K, Regan L, Spring J, et al. A further look at infection at the time of therapeutic abortion. Eur J Obstet Gynecol Reprod Biol 1990; 37: 231-236.

Blackwell AL, Thomas PD, Wareham K, at. He lower genital tract in women attending for TOP. Lancet 1993; 342: 206-210.

Smith N, Neilson MR, Hammond J, et al. Screening for lower genital tract infections in women presenting for TOP. Int J STD AIDS 1994; 5: 212-213.

Sewart P. Prevalence of potential pathogens in the cervical canal prior to termination of pregnancy. Br Med J 1992: 304:1479.

Standards for partner notification. In: Clinical Guidelines 'Genital Chlamydial Infection'. Central Audit Group in Genitourinary Medicine, November 1997.

Duynhoven van YTHP, Schop WA, et al. Patient referral outcome in gonorrhoea and chlamydial infections. Sex Trans Inf 1998; 74:323-330.

Cowan FM, French R, Johnson AM. The role and effectiveness of partner notification in STD control: a review. Genitourin Med 1996; 72: 247-252.

3 Kissinger P, Brown R, Reed K, et al. Effectiveness of patient delivered partner medication for preventing recurrent Chlamydia trachomatis. Sex Trans Inf 1998; 74:331-333. 